\title{
THE ZEROS OF HOLOMORPHIC FUNCTIONS IN STRICTLY PSEUDOCONVEX DOMAINS
}

\author{
BY

\section{LAWRENCE GRUMAN}

ABSTRACT. We determine a sufficient condition on a positive divisor in certain strictly pseudoconvex domains in $C^{n}$ such that there exists a function in the Nevanlinna class which determines the divisor.

For $D$ the unit disc in $\mathbf{C}$, the relationship between the zeros of holomorphic functions (resp. the zeros and poles of meromorphic functions) and the growth of holomorphic (resp. meromorphic) functions with the same zeros (resp. zeros and poles) has been extensively studied. A holomorphic function $f$ is in the Nevanlinna class $N(D)$ if and only if

$$
\lim _{r \rightarrow 1} \int_{0}^{2 \pi} \log ^{+}\left|f\left(r e^{i \theta}\right)\right| d \theta<\infty .
$$

A meromorphic function $g$ is said to be of bounded characteristic if

$$
\begin{gathered}
\int_{0}^{1} \frac{n(t)}{t} d t<\infty \text { where } n(t) \text { counts the poles of } g ; \\
\qquad \lim _{r \rightarrow 1} \int_{0}^{2 \pi} \log ^{+}\left|g\left(r e^{i \theta}\right)\right| d \theta<\infty .
\end{gathered}
$$

If $f \in N(D)$, it is well known that $f$ has nontangential limit values almost everywhere on $\partial D$ and that its zeros $\dot{a}_{n}$ satisfy the Blaschke condition $\sum_{n=1}^{\infty}\left(1-\left|a_{n}\right|\right)$ $<\infty$. This implies that there exists a bounded holomorphic function $\widetilde{f}$ with the same zeros as $f$. If $g$ is a meromorphic function of bounded characteristic, then $g=f / h$, where $f, h \in N(D)$ and hence $g$ has nontangential limit values almost everywhere on the unit circle. Very little is known about related results in domains in $\mathbf{C}^{\boldsymbol{n}}$. We propose to study this question.

Let $\delta$ be a positive divisor in a domain of holomorphy $D \subset \mathbf{C}^{n} ; \delta=\left(X_{j}, d_{j}\right)$ where $X=\bigcup_{j} X_{j}$ is an analytic subvariety of codimension 1 with irreducible branches $X_{i}$ and the $d_{j}$ 's are positive integers (cf. Stoll [17]). If the second Cech cohomology group with integer coefficients is trivial $\left(H^{2}(D, Z)=0\right)$, then there exists a holomorphic function $f$ in $D$ such that $f$ determines the divisor $\delta$-that is,

Received by the editors March 25, 1974.

AMS (MOS) subject classifications (1970). Primary 32A10, 32 A20.

Key words and phrases. Nevanlinna class, Nevanlinna characteristic.

Copyright $\odot$ 1975. American Mathematical Society 
$f$ vanishes on $X_{j}$ to order $d_{j}$ and $f(z) \neq 0$ for $z \notin X$ (cf. Hörmander [5, p. 181]). If we set $\mu=\Delta \log |f| / 2 \pi$ taken in the sense of a distribution, then $\mu$ is a positive measure in $D$ which measures the "area" of the divisor $\delta$ (cf. Lelong [9], [10], [11]). We say that the divisor $\delta$ has finite area in $D$ if $\int_{D} d \mu<\infty$. If $\delta$ is a meromorphic divisor in $D$, then $\delta=\delta^{+}-\delta^{-}$, where $\delta^{+}$and $\delta^{-}$are two positive divisors. We say that $\delta$ is of finite area in $D$ if both $\delta^{+}$and $\delta^{-}$are of finite area in $D$.

A bounded domain $D \subset \mathbf{C}^{n}$ is strictly pseudoconvex if there exists a twice continuously differentiable plurisubharmonic function $\rho(z)$ defined in a neighborhood $N$ of $\partial D$ such that

$$
N \cap D=\{z: \rho(z)<0\}, \quad \partial D=\{z: \rho(z)=0\},
$$

and the complex Hessian of $\rho, \Sigma_{j, k}\left(\partial^{2} \rho / \partial z_{j} \partial \bar{z}_{k}\right)$ is positive definite in $N$. We also assume that $\nabla \rho \neq 0$ in a neighborhood of $\partial D$ (cf. Henkin [4]).

The Nevanlinna class of $D, N(D)$, is defined to be the set of holomorphic functions $f$ defined in $D$ such that

$$
\varlimsup_{\epsilon \rightarrow 0^{+}} \int_{\partial D_{\epsilon}} \log ^{+}|f(z)| d S_{\epsilon}(z)<\infty
$$

where $D_{\epsilon}$ is the strictly pseudoconvex domain determined by $\rho(z)+\epsilon$ (for $|\epsilon|$ small) and $d S_{\epsilon}$ is the Lebesgue measure of $\partial D_{\epsilon}$. Such functions possess nontangential limit values (different from zero) almost everywhere on $\partial D([2],[16, \mathrm{p}$. 48]). We show

THEOREM. If $\delta$ is a positive divisor of finite area in a strictly pseudoconvex domain $D$ with $\mathrm{C}^{3}$ boundary and $H^{2}(D, \mathrm{Z})=0$ then there exists a function $f \in N(D)$ such that $f$ determines the divisor $\delta$.

If $D$ is starlike, then we need only assume $C^{2}$ boundary, and it is likely that the theorem is true for all strictly pseudoconvex domains with $C^{2}$ boundary (such that $H^{2}(D, Z)=0$ ). In [7], Laville showed that under the above hypotheses, if $D$ is starlike, then there exists an $f$ which determines $\delta$ such that $\log |f| \in$ $L^{1}(d \lambda)$, where $d \lambda$ is the Lebesgue measure in $D$.

A meromorphic function $g$ is of bounded characteristic in $D$ if

$$
\varlimsup_{\epsilon \rightarrow 0^{+}} \int_{\partial D_{\epsilon}} \log ^{+}|g(z)| d S_{\epsilon}(z)<\infty .
$$

(ii) $g=f / h$ for two holomorphic functions and $\int_{D}(\Delta \log |h|) \rho d \lambda<\infty$.

We show

Corollary. Let $D$ be as in the Theorem and let $g$ be a meromorphic func- 
tion of bounded characteristic in $D$ such that the divisor $\delta$ determined by $g$ is of finite area in $D$. Then $g$ can be written as the quotient of two functions in $N(D)$ and hence $g$ has nontangential limit values almost everywhere on $\partial D$.

This corrects the statement of the Theorem as incorrectly announced in [1]. For related results on the Nevanlinna characteristic using different scales of growth, the reader is referred to [2] by the author and to Mueller [12].

If $\delta$ is of finite area, there may well not exist a bounded function which vanishes on $\delta$ as the following simple argument shows. Let $B=\left\{z: \sum_{k=1}^{n}\left|z_{k}\right|^{2}<1\right\}$ be the unit ball in $\mathbf{C}^{n}$ and for some sequence $\left\{a_{j}\right\}$, let $X_{j}=\left\{z \in B: z_{1}=a_{j}\right\}$. Let $\delta=\left(X_{j}, 1\right)$. The "area" of $X_{j}$ is just $C_{n}\left[\left(1-\left|a_{j}\right|^{2}\right)^{1 / 2}\right]^{(2 n-2)}$. Thus, if we choose $\left\{a_{j}\right\}$ such that $\Sigma_{j}\left(1-\left|a_{j}\right|\right)^{n-1}$ converges, but $\Sigma_{j}\left(1-\left|a_{j}\right|\right)$ diverges, then $\delta$ is of finite area in $B$, but there exists no bounded function which determines $\delta$ since for $z_{2}=\cdots=$ $z_{n}=0$, the sequence $\left\{a_{j}\right\}$ does not satisfy the Blaschke condition.

The ideas we use here were first employed by Skoda [15] to resolve the second Cousin problem in $\mathbf{C}^{n}$ with growth conditions. He used the Hörmander $L^{2}$ resolution of the $\bar{\partial}$-Neumann operator with weight functions [5]. We use the resolution in strictly pseudoconvex domains by use of a kernel function as developed by Ramírez de Arellano [14], Henkin [3], Kerzman [6], and Ovrelid [13], which allows for finer results.

1. Resolution of the $\bar{\partial} \bar{\partial}$ problem. We denote the differential operators $\partial, \bar{\partial}$ and $d$ by

$$
\partial=\sum_{j=1}^{n} \frac{\partial}{\partial z_{j}} d z_{j}, \quad \bar{\partial}=\sum_{j=1}^{n} \frac{\partial}{\partial \bar{z}_{j}} d \bar{z}_{j}, \quad d=\partial+\bar{\partial} .
$$

If $\alpha$ is a differential form with functions for coefficients, we define $|\alpha|=$ $\Sigma_{p, q}\left|\alpha_{p, q}\right|$ where $\alpha_{p, q}$ are the coefficients.

We begin by assuming that $D$ is a bounded starlike domain with the origin as starcenter and that $\delta$ is defined in a neighborhood of $\bar{D}$. We follow closely the development of Skoda [15].

We choose a function $\beta \in C_{0}^{\infty}(B(0,1))$ such that $\beta$ depends only on $\|z\|$, $0 \leqslant \beta \leqslant 1$, and $\int \beta d \lambda=1$. We set $\beta_{\epsilon}(z)=\epsilon^{-2 n} \beta(z / \epsilon)$. Let $f$ be any holomorphic function which determines $\delta$ in a neighborhood of $\bar{D}$. We define $V^{\epsilon}(z)=$ $\log |f| * \beta_{\epsilon}$ which is $C^{\infty}$ and plurisubharmonic in $D$ for $\epsilon$ sufficiently small. Let

$$
\omega_{\delta}^{\epsilon}=i \partial \bar{\partial} V^{\epsilon}=i \sum_{j, k} \theta_{j k}^{\epsilon} d z_{j} \wedge d \bar{z}_{k}, \quad \text { where } \theta_{j k}^{\epsilon}=\frac{\partial^{2} V^{\epsilon}}{\partial z_{j} \partial \bar{z}_{k}} .
$$

This defines a positive closed current in $D$ (cf. Lelong [11]) which depends on $\delta$ and $\epsilon$ but not on $f$. We will in general drop the subscript $\delta$ when there is no 
chance of confusion. We have the relations:

(i) $\theta_{j j}^{\epsilon} \geqslant 0$,

(ii) $\theta_{j k}^{\epsilon}=\bar{\theta}_{k j}^{\epsilon}$,

(iii) $\left|\theta_{j k}^{\epsilon}\right| \leqslant \theta_{j j}^{\epsilon}+\theta_{k k}^{\epsilon} \leqslant \mu^{\epsilon}$ where $\mu^{\epsilon}=\sum_{j=1}^{n} \theta_{j j}^{\epsilon}$,

(iv) $\theta_{j k}^{\epsilon}=\theta_{j k} * \beta_{\epsilon}$, where $\theta_{j k}$ is the measure $\partial^{2} V / \partial z_{j} \partial \bar{z}_{k}$ and supp $\theta_{j k} \subset X$. If we write

$$
\begin{aligned}
& \nu_{1}^{\epsilon}=\sum_{j=1}^{n}\left[\sum_{k=1}^{n} \bar{z}_{k} \int_{0}^{1} t \theta_{j k}^{\epsilon}(t z) d t\right] d z_{j}, \\
& \nu_{2}^{\epsilon}=\sum_{j=1}^{n}\left[\sum_{k=1}^{n} z_{k} \int_{0}^{1} t \theta_{k j}^{\epsilon}(t z) d t\right] d \bar{z}_{j},
\end{aligned}
$$

then $\bar{\nu}_{1}^{\epsilon}=\nu_{2}^{\epsilon}, \bar{\partial} \nu_{2}^{\epsilon}=\partial \nu_{1}^{\epsilon}=0$ and $\omega^{\epsilon}=i\left(\partial \nu_{2}^{\epsilon}-\bar{\partial} \nu_{1}^{\epsilon}\right)$.

We shall find a function $u_{2}^{\epsilon}$ such that $\bar{\partial} u_{2}^{\epsilon}=\nu_{2}^{\epsilon}$ in $D$. Then setting $u^{\epsilon}=$ $u_{2}^{\epsilon}+\bar{u}_{2}^{\epsilon}$, we have $i \partial \bar{\partial} u^{\epsilon}=\omega^{\epsilon}$ so $u^{\epsilon}$ is plurisubharmonic and defines the current $\omega^{\epsilon}$.

Let $D_{r}=\{r z: z \in D\}$ and let $S_{r}=\partial D_{r}$. If we set $\sigma^{\epsilon}(r)=\int_{D_{r}} \mu^{\epsilon}(z) d \lambda(z)$, then it follows from (iii) and (1) that

$$
\int_{S_{r}}\left|\nu_{i}^{\epsilon}\right| d S \leqslant C \sigma^{\epsilon}(r), \quad i=1,2 .
$$

2. The kernel for $\bar{\partial} f=\nu_{2}$. Let $g_{j}(z, w), j=1, \ldots, n$, be $C^{1}$ functions defined on a neighborhood of $\bar{D} \times \bar{D}$ such that $g_{j}(z, w)=\left(\bar{z}_{j}-\bar{w}_{j}\right)$ for $\|z-w\|$ small and such that $G(z, w)=\sum_{j=1}^{n}\left(z_{j}-w_{j}\right) g_{j}$ satisfies

(i) $\operatorname{Re} G(z, w)>0$ for $z \in \partial D, w \in D$,

(ii) $|G(z, w)| \neq 0$ for $z \neq w, w \in D, z \in \bar{D}$,

(iii) for $w \in D$ fixed, $G(z, w)$ is holomorphic in $w$ for $z$ near $\partial D$.

If we set

$$
K(z, w)=C_{n} \sum_{j=1}^{n} \frac{(-1)^{j+1}}{[G(z, w)]^{n}} g_{j} \bigwedge_{k \neq j} \bar{\partial} g_{k} \bigwedge_{k=1}^{n} d z_{k}
$$

with $C_{n}=(-1)^{n(n-1) / 2}(n-1) ! /(2 \pi i)^{n}$ and if $\tau$ is a $(0,1)$ form such that $\bar{\partial} \tau=0$, then

$$
u_{\tau}(w)=\int_{D} K(z, w) \wedge \tau(z)
$$

defines a function in $D$ such that $\bar{\partial} u_{\tau}(w)=\tau(w)$ (cf. Kerzman [6] and Ovrelid [13]).

At this point, as in [7], we could use the estimates of Kerzman and Ovrelid 
to find a holomorphic function $f$ such that $\log |f| \in L^{1}(d \lambda)$, but these estimates are too crude since the main contribution comes from the zeros of $f$, where $\log |f|$ is near $-\infty$. Thus, we want to separate out that part of the integral which gives a negative value. To do this, we introduce the form $M(z, w)$ below. The constant $C_{n}^{\prime}$ is so chosen as to eliminate integration on the divisor near the point $w$.

Let $\beta=\partial \bar{\partial}\|z\|^{2}=\Sigma_{j=1}^{n} d z_{j} \wedge d \bar{z}_{j}$ and let $\beta_{m}=\beta^{m} / m !$. We define

$$
M(z, w)=\frac{-C_{n}^{\prime}}{[G(z, w)]^{n-1}} \beta_{n-1}
$$

with $C_{n}^{\prime}=(-1)^{n}(n-2) ! /(2 \pi i)^{n}$. Then by Stoke's Theorem, it follows that

$$
\begin{aligned}
C_{n}^{\prime} \int_{D} \partial_{z} M(z, w) \wedge \tau(z) & +C_{n}^{\prime} \int_{D} \frac{-1}{[G(z, w)]^{n-1}} \beta_{n-1} \wedge \partial \tau(z) \\
& =C_{n}^{\prime} \int_{\partial D} M(z, w) \wedge \tau(z)=f_{\tau}(w)
\end{aligned}
$$

and since $G(z, w)$ is holomorphic for $w \in D, z \in \partial D, f_{\tau}+\bar{f}_{\tau}$ is pluriharmonic in $D$.

3. Functions of Nevanlinna class in $D$. We begin by proving the following specific case of the theorem, and we then reduce the general case to the specific case by a patching argument.

LemmA. Let $D$ be a strictly convex domain given by a $C^{2}$ norm $p(z)$ (i.e. $D=\{z: p(z)<1\})$. If $\delta$ is a divisor of finite area in $D$, then there exists a function $f \in N(D)$ which defines $\delta$.

Proof. Let $\psi_{\eta}(\dot{r}), r \in R$, be defined by

$$
\psi_{\eta}(r)=\left\{\begin{array}{l}
1 \text { for } r \leqslant 1 / 4 \eta, \\
0 \text { for } r \geqslant 1 / 2 \eta, \\
1-\frac{(r-1 / 4 \eta)}{1 / 4 \eta} \text { for } 1 / 4 \eta<r<1 / 2 \eta .
\end{array}\right.
$$

We set $\phi_{\eta}(z, w)=\psi_{\eta}(\rho(z) / \rho(w))$ where $\rho(z)=p(z)-1$, and we let $\bar{\phi}^{\eta}(z, w)=$ $\psi_{\eta}^{\prime}(\rho(z) / \rho(w))$; we set $g_{j}^{\eta}=\left(\bar{z}_{j}-\bar{w}_{j}\right)\left(1-\phi^{\eta}\right)+\xi_{j} \phi^{\eta}$ in (3), where $\xi_{j}=\partial \rho / \partial z_{j}$.

Since the solution $u_{\tau}(\dot{w})$ given by (4) is independent of the patching function $\phi[6, p .341]$, if $\tau$ has $C^{1}$ coefficients, we need only that $\phi$ be piecewise differentiable as above, and furthermore, $\tau$ is independent of $\eta$.

We begin by assuming that $\delta$ is a divisor in a neighborhood of $\bar{D}$ and we define $\nu_{2}^{\epsilon}$ as in $\S 1$, where $\epsilon$ is small enough so that $V_{\epsilon}$ is plurisubharmonic in D. Let 


$$
\begin{aligned}
u_{\epsilon}(w)= & \int_{D}\left[K(z, w)+\partial_{z} M(z, w)\right] \wedge \nu_{2}^{\epsilon} \\
& +C_{n}^{\prime} \int_{D} \frac{-1}{[G(z, w)]^{n-1}} \beta_{n-1} \wedge \partial v_{2}^{\epsilon} \\
= & I_{1}+I_{2} .
\end{aligned}
$$

Then $i \partial \bar{\partial} u_{\epsilon}(w)=\omega^{\epsilon}$. If we set $u_{\epsilon}^{+}=\sup \left(0, \operatorname{Re} u_{\epsilon}\right)$ then we shall show that $\int_{\partial D} u_{\epsilon}^{+} \leqslant M$ independent of $\epsilon$ for $\epsilon$ sufficiently small.

First we note that for $\phi_{\eta}(z, w)=0,-1 /[G(z, w)]^{n-1}<0$ and

$$
\operatorname{Re}\left\{(-i)^{n} \beta_{n-1} \wedge \partial \nu_{2}^{\epsilon}\right\}=\operatorname{Re}\left\{(-i)^{n} \beta_{n-1} \wedge 1 / 2 \omega^{\epsilon}\right\}=\mu^{\epsilon} d \lambda / 2^{n}
$$

(cf. Lelong [9], [10]). Thus, since $1 /|G(z, w)| \leqslant C /\|z-w\|^{2 n-2}[6, \S 3]$, if we choose $\eta$ sufficiently small (depending on $\epsilon$ ), $\operatorname{Re} I_{2} \leqslant 1$.

Now

$$
\partial M(z, w)=\frac{(n-1) C_{n}^{\prime}}{[G(z, w)]^{n}}\left[\sum_{j} g_{j}^{n} d z_{j} \wedge \beta_{n-1}+\left(z_{j}-w_{j}\right) \partial g_{j}^{n} \wedge \beta_{n-1}\right]
$$

and

$$
\begin{aligned}
K(z, w)= & \frac{C_{n}}{[G(z, w)]^{n}}\left\{\sum _ { j } ( - 1 ) ^ { j + 1 } g _ { j } ^ { n } \bigwedge _ { k \neq j } \left[d \bar{z}_{k}\left(1-\phi_{\eta}\right)+\partial \xi_{k} \phi\right.\right. \\
& \left.\left.+\left(\xi_{k}-\left(\bar{z}_{k}-w_{k}\right)\right) \bar{\partial} \phi^{n}\right] \bigwedge_{k=1}^{n} d z_{k}\right\} \\
= & \frac{C_{n}}{[G(z, w)]^{n}}\left\{\sum_{j}(-1)^{j+1} g_{j}^{n} \bigwedge_{k \neq j} d \bar{z}_{k} \bigwedge_{k=1}^{n} d z_{k}+R\right\}
\end{aligned}
$$

where $R$ is an $(n, n-1)$ form for which each coefficient has a factor of the form $\left(\bar{z}_{k}-\bar{w}_{k}\right),\left(\phi^{\eta}\right)^{2}$, or $\left(\bar{z}_{k}-\bar{w}_{k}\right) \bar{\phi}^{\eta}$. No term involves more than one factor of the form $\psi^{\prime}$ since $\bar{\partial} \phi^{\eta} \wedge \bar{\partial} \phi^{\eta} \equiv 0$ (cf. [6, p. 350]).

For fixed $w$, we let $D_{w}^{\prime}=\{z: \rho(z) / \rho(w) \leqslant 1 / 2 \eta\}$ and $S_{w}=\left\{w^{\prime}: \rho\left(w^{\prime}\right)=\rho(w)\right\}$. We set $N_{\epsilon}(\omega)=\int_{0}^{1} \mu_{\epsilon}(t \omega) d t$ for $\omega \in \partial D$. Then if $\delta$ has finite area in $D$, $\int_{\partial D} N_{\epsilon}(\omega) d S<C$ independent of $\epsilon$ for $\epsilon$ small, and by (1) and (iii) of $\S 1$,

$$
\left|v_{2}^{\epsilon}(z)\right| \leqslant N_{\epsilon}(z / p(z)), \quad z \neq 0 .
$$

We have shown that 


$$
\begin{gathered}
u_{\epsilon}^{+}(w) \leqslant \operatorname{Re}\left\{\int_{D_{w}^{\prime}}\left[K(z, w)+\partial_{z} M(z, w)\right] \wedge \nu_{2}^{\epsilon}(z)\right. \\
\left.+\frac{C_{n}(-i)^{n}}{2^{n}} \int_{D_{w}^{\prime}} \frac{-1}{[G(z, w)]^{n-1}} \beta_{n-1} \wedge \partial \nu_{2}\right\} \\
\leqslant C\left\{\int_{D_{w}^{\prime}} \frac{|z-w|\left(1+\left|\bar{\phi}^{\eta}\right|\right)+\left(\phi^{\eta}\right)^{2}}{|G(z, w)|^{n}}\left|\nu_{2}^{\epsilon}(z)\right| d \lambda\right\}+1 .
\end{gathered}
$$

We shall estimate a term of the form

$$
\int_{S_{w}} \int_{D_{w}^{\prime}} \frac{\left|\overline{z_{k}}-\bar{w}_{k}^{\prime}\right|}{|G(z, w)|^{n}}\left|\bar{\phi}^{\eta}\right|\left|\nu_{2}^{\epsilon}(z)\right| d \lambda(z) d S\left(w^{\prime}\right)=\int_{S_{w}} A\left(w^{\prime}\right) d s\left(w^{\prime}\right)
$$

The others are handled in a similar (perhaps simpler) manner with straightforward modifications.

We choose as local coordinates on $S_{w}, v=\left[v_{1}, v^{\prime}\right], v_{1}=I_{m}\left\langle\xi, z-w^{\prime}\right\rangle, v^{\prime} \in$ $\mathbf{R}^{2 n-2}$ and we let $u_{1}=-\rho(z)$. If $r=\left\|z-w^{\prime}\right\|, \alpha=\eta|\rho(w) / 2|$, then there exist constants $c_{1}$ and $c_{2}$ such that

$$
c_{1}\left[\alpha+\left|v^{\prime}\right|+\left|v_{1}\right|\right] \leqslant r \leqslant c_{2}\left[\alpha+\left|v^{\prime}\right|+\left|v_{1}\right|\right]
$$

(see $[6],[13, \S 5])$. Then

$$
\begin{aligned}
\int_{S_{w}} A\left(w^{\prime}\right) d S\left(w^{\prime}\right) & +\frac{2}{\alpha} \int_{\partial D} \int_{S_{w}} \int_{0}^{\alpha / 2} \frac{r N_{\epsilon}(\omega) d u_{1} d S\left(w^{\prime}\right) d S(\omega)}{\left[r^{2}+\phi\left|v_{1}\right|\right]^{n}} \\
& \leqslant \frac{C_{1}}{\alpha} \int_{\partial D} \int_{S_{w}} \int_{0}^{\alpha / 2} \frac{N_{\epsilon}(\omega) d u_{1} d S\left(w^{\prime}\right) d S(\omega)}{\left[|v|^{2}+\alpha^{2}+\left|v_{1}\right|^{2}+\phi\left|v_{1}\right|\right]^{n-1 / 2}}
\end{aligned}
$$

Since $\phi^{\eta}$ depends only on $u_{1}$, for fixed $w^{\prime}$, we divide the interval $[0, \alpha / 2]$ onto two subintervals, one on which $\phi^{\eta} \leqslant|v|^{1 / 2}$, of length at most $|v|^{1 / 2} \alpha$, and one on which $\phi^{\eta} \geqslant|v|^{1 / 2}$, of length at most $\alpha$. Thus

$$
\begin{aligned}
\int_{S_{w}} A\left(w^{\prime}\right) d S\left(w^{\prime}\right) \leqslant C_{1}\left\{\int_{\partial D}\right. & \int_{S_{w}} \frac{N_{\epsilon}(\omega) d S\left(w^{\prime}\right) d S(\omega)}{\left[|v|^{2}+\alpha^{2}+\phi\left|v_{1}\right|\right]^{n-3 / 4}} \\
& \left.+\int_{\partial D} \int_{S_{w}} \frac{N_{\epsilon}(\omega) d S\left(w^{\prime}\right) d S(\omega)}{\left[|v|^{2}+\alpha^{2}+\left|v_{1}\right||v|^{1 / 2}\right]^{n-1 / 2}}\right\}
\end{aligned}
$$

Now 


$$
\int_{S_{w}} \frac{d S\left(w^{\prime}\right)}{\left[|v|^{2}+\phi\left|v_{1}\right|\right]^{n-3 / 4}} \leqslant C_{2} \int \frac{|v|^{2 n-2}}{|v|^{2 n-3 / 2}} d v \leqslant K_{1}
$$

and

$$
\begin{aligned}
\int_{S_{w}} \frac{d S\left(w^{\prime}\right)}{\left[|v|^{2}+\left|v_{1}\right||v|^{1 / 2}\right]^{n-1 / 2}} & \leqslant C_{2}^{\prime} \int \frac{d v_{1}\left|v^{\prime}\right|^{2 n-3} d v^{\prime}}{\left[\left|v^{\prime}\right|^{2}+\left|v_{1}\right|\left|v^{\prime}\right|^{1 / 2}\right]^{n-1 / 2}} \\
& \leqslant C_{2}^{\prime} \int \frac{1}{\left|v^{\prime}\right|^{1 / 2}} \frac{\left|v^{\prime}\right|^{2 n-3}}{\left|v^{\prime}\right|^{2(n-3 / 2)}} d v^{\prime} \\
& \leqslant \int \frac{d v^{\prime}}{\left|v^{\prime}\right|^{1 / 2}} \leqslant K_{2}
\end{aligned}
$$

so $\int_{S_{w}} A\left(w^{\prime}\right) d S\left(w^{\prime}\right) \leqslant C \cdot \sup \left(K_{1}, K_{2}\right)$. After computing the other terms in a similar fashion, we see that

$$
\int_{S_{w}} u_{\epsilon}^{+}\left(w^{\prime}\right) d S\left(w^{\prime}\right) \leqslant M \quad \text { independent of } w \text { and } \epsilon .
$$

Let $f$ be any holomorphic function which defines the divisor $\delta$ in $D$, and form $V^{\epsilon}=\log |f| * \beta_{\epsilon}$ as in $\S 1$. Since $\log |f|$ is plurisubharmonic, it is locally integrable, so $V^{\epsilon}$ is uniformally locally integrable for $\epsilon$ small enough. Set $H_{\epsilon}(z)=$ $u^{\epsilon}-V^{\epsilon}$. Then for any open set $U$ with compact closure in $D, H_{\epsilon}$ is pluriharmonic for $\epsilon$ sufficiently small. Let $\omega(z) \in C_{0}^{\infty}(D)$ be such that $0 \leqslant \omega(z) \leqslant 1$ and $\omega(z) \equiv 1$ on $U$. Then for $z \in U$,

$$
V^{\epsilon}(z)=\frac{(n-2) !}{(2 \pi)^{n}} \int \frac{-1}{\|z-w\|^{2 n-2}} \Delta\left[\omega V^{\epsilon}\right] d \lambda(w) .
$$

We integrate by parts those terms involving $\left(\partial \omega / \partial z_{i}\right)\left(\partial V^{\epsilon} / \partial \bar{z}_{i}\right)$ or $\left(\partial \omega / \partial \bar{z}_{i}\right)\left(\partial V^{\epsilon} / \partial z_{i}\right)$ to get, for instance

$$
\int \frac{\partial}{\partial \bar{z}_{i}}\left[\frac{-1}{\|z-w\|^{2 n-2}} \frac{\partial \omega}{\partial \bar{z}_{i}}\right] V^{\epsilon} d \lambda(w) .
$$

Thus, it follows from the estimates that we have made that $H_{\epsilon}(z)$ is bounded in absolute value in $U$ for $\epsilon$ sufficiently small.

It follows from the Poisson Integral Formula that the family $H_{\epsilon}(z)$ is equicontinuous and hence from the Arzela-Ascoli Theorem, we can choose a sequence $\epsilon_{i} \rightarrow 0$ such that $H_{\epsilon_{i}}(z)$ converges to a function $H(z)$ uniformly on compact subsets so that $H(z)$ is pluriharmonic in $D$. Let $u(z)=H(z)+\log |f|$. Then $i \partial \bar{\partial} u(z)$ $=\omega$ on the sense of a distribution and $\lim _{r \rightarrow 1} \int_{\partial D} u(r z)^{+} d S \leqslant M$. 
In fact, we have

$$
\int_{\partial D}\|\log \mid f(r \omega)\| d S(\omega) \leqslant M^{n}
$$

independent of $r$. To see this, we let $q \in D$ be such that $\log |f(q)|>M^{\prime}>-\infty$. Then $\int_{\partial D} \log |f(r w)| P(q, w) d S(w) \geqslant M^{\prime}$, where $P(\cdot, w)$ is the Poisson kernel for D. This proves (6). Q.E.D.

REMARK. By using the kernel of Ovrelid [13], in the very same manner, one can show that for a starlike strictly pseudoconvex domain, one need only assume $C^{2}$ boundary (locally, the kernel is the one we have used above). To piece the solutions together in the general case, however, we need to assume $\mathrm{C}^{3}$ boundary below.

Proof of Theorem. Let $\xi \in \partial D$ where $D$ is a strictly pseudoconvex domain with $C^{3}$ boundary. Then there exist a neighborhood $U_{\xi}$ of $\xi$ and a biholomorphic map $F$ of $U_{\xi}$ onto a neighborhood $Y_{\xi}$ of the origin in $C^{n}$ such that $F(\xi)=0$ and $\rho^{\prime}=\rho \circ F^{-1}$ is strictly convex-that is

$$
\sum_{i, j=1}^{2 n} \frac{\partial^{2} \rho^{\prime}}{\partial x_{i} \partial x_{j}} t_{i} t_{j}>0
$$

for $x_{i}$ the underlying real coordinates. We choose $F$ so that $\rho^{\prime}$ satisfies $\nabla \rho^{\prime}(0)=$ $(\partial \rho / \partial w, 0, \ldots, 0)$. Let $\rho^{\prime \prime}(\xi, w)=\rho^{\prime}(w)+\phi(|w|)$ where $\phi(t)$ is a $C^{\infty}$ nonnegative increasing convex function of the real variable $t$ and $\phi \equiv 0$ in a neighborhood of the origin. If $D^{\prime \prime}(\xi)=\left\{w: \rho^{\prime \prime}(\xi, w)<0\right\}$; than for appropriate $\phi, D^{\prime \prime}(\xi) \subset Y_{\xi}$ and $D^{\prime \prime}(\xi)$ is strictly convex with $C^{3}$ boundary. By the lemma, there exists $f_{\xi}^{\prime} \in$ $N\left(D^{\prime \prime}(\xi)\right)$ such that $f_{\xi}^{\prime}$ determines the divisor $\delta^{\prime}=\left(F\left(X_{j}\right), d_{j}\right)$ in $D^{\prime \prime}(\xi)$. We let $f_{\xi}=f_{\xi}^{\prime} \circ F$ and $G(\xi)=F^{-1}\left(D^{\prime \prime}(\xi)\right)$. Then $f_{\xi}$ determines $\delta$ in $G(\xi)$ and $f_{\xi} \in$ $N(G(\xi))$.

We choose a finite number of domains $G_{i}$ and functions $f_{i}$ such that:

(i) $\cup G_{i}=D$;

(ii) if $z \in \bar{D}$, then there exists an $i$ such that $d\left(z, C \bar{G}_{i} \cap \bar{D}\right)>\eta>0$ independent of $z$;

(iii) $f_{i}$ determines $\delta$ in $G_{i}$ and $f_{i} \in N\left(G_{i}\right)$.

We now cover $\bar{D}$ by a finite number of balls $B_{j}=B\left(z_{j} ; r_{j}\right)$ such that, setting $D_{j}(r)=B\left(z_{j}, r\right) \cap D$, we have

(a) $D_{j}\left(2 r_{j}\right) \subset G_{i}$ for some $i$,

(b) $D_{j}\left(2 r_{j}\right) \cap D_{k}\left(2 r_{k}\right)$ is simply connected.

We choose a partition of unity $\left\{\phi_{j}, \phi_{0}\right\}$ for $B\left(z_{j}, 2 r_{j}\right), D_{0}=\mathrm{C} \bar{D}$. We let $f_{j}=$ $\left.f_{i}\right|_{D_{j}\left(2 r_{j}\right)}$ for some $i$ which satisfies (a). 
Let $g_{j k}=\log f_{j}-\log f_{k}$ in $D_{j}\left(2 r_{j}\right) \cap D_{k}\left(2 r_{k}\right)$, where we can choose the principal branch of the logarithm since the domain is simply connected and $f_{j} / f_{k} \neq 0, f_{k} / f_{j} \neq 0$. Let $h_{j}=\Sigma_{k \neq j} g_{j k} \phi_{k}$ in $D_{j}\left(2 r_{j}\right)$. Then $u=\bar{\partial} h_{j}$ is globally defined.

It follows from the construction in $[13, \S 5]$ that we can choose $K(z, w)$ in (3) so that $|K(z, w)|<C$ for $|z-w| \geqslant \min \left(r_{j} / 4\right)$.

We let $S_{j}^{\delta}=\left\{w \in D_{j}\left(r_{j}\right): \rho(w)=-\delta\right\}$. Then for $r \geqslant 3 r_{j} / 2$,

$$
\begin{aligned}
v(w) & =\int_{D} K(z, w) \wedge u(z)=\int_{C D_{j}(r)} K(z, w) \wedge u(z)+\int_{D_{j}(r)} K(z, w) \wedge u(z) \\
& =v_{1}(w, r)+v_{2}(w, r), \\
v(w) & =\frac{2}{r_{j}}\left\{\int_{3 r_{j} / 2}^{2 r_{j}} v_{1}(w, t) d t+\int_{3 r_{j} / 2}^{2 r_{j}} v_{2}(w, t) d t\right\}=v_{j}^{\prime}(w)+v_{2}^{\prime}(w) .
\end{aligned}
$$

Since $|K(z, w)|$ is bounded for $z \in C D_{j}(r), r \geqslant 3 r_{j} / 2, w \in D_{j}\left(r_{j}\right)$ and since $|u(z)|$ is integrable by (6), $\left|v_{1}^{\prime}\left(w^{\prime}\right)\right| \leqslant K_{1}(j)$ for $w^{\prime} \in D_{j}\left(r_{j}\right)$. we have

Since $u=\bar{\partial} h_{j}$ in $D_{j}\left(2 r_{j}\right)$, by applying Stoke's Theorem (as in $[6$, p. 340])

$$
\begin{aligned}
\int_{D_{j}(r)} K(z, w) \wedge \bar{\partial} h_{j} & =\int_{D_{j}(r)} \bar{\partial}\left[h_{j} K(z, w)\right]=\int_{D_{j}(r) \backslash\{w\}} d\left[h_{j}(z) K(z, w)\right] \\
& =h_{j}(w)+\int_{\partial D_{j}(r)} h_{j}(z) K(z, w)
\end{aligned}
$$

so

$$
v_{2}^{\prime}(w)=\frac{2}{r_{j}} h_{j}(w)+\frac{2}{r_{j}} \int_{3 r_{j} / 2}^{2 r_{j}}\left(\int_{\partial D_{j}(t)} h_{j}(z) K(z, w)\right) d t=v_{3}(w)+v_{4}(w) .
$$

Let $A_{j}(r)=\left\{z: z \in \partial D_{j}(r) \cap \partial D\right\}$ and let $B_{j}=\left\{z: z \in \partial D_{j} \cap D\right.$ for some $r$, $3 r_{j} / 2 \leqslant r \leqslant 2 r_{j}$. Let $\alpha_{j}$ be in $C_{0}^{\infty}\left(B_{j}\left(z_{j}: 5 r_{j} / 4\right)\right)$ such that $\alpha_{j} \equiv 1$ on $B_{j}\left(z_{j}, r_{j}\right)$. Then

$$
\begin{aligned}
\left|v_{4}(w)\right| \leqslant & \frac{2}{r_{j}} \int_{B_{j}}\left|h_{j}(z)\right||K(z, w)|+\frac{2}{r_{j}}\left|\int_{A_{j}(t)}\left(1-\alpha_{j}\right) h_{j}(z) K(z, w) d t\right| \\
& +\left|\int_{A_{j}\left(3 r_{j} / 2\right)} \alpha_{j} h_{j}(z) K(z, w)\right| \leqslant v_{5}(w)+v_{6}(w)+v_{7}(w) .
\end{aligned}
$$

Now $\left|v_{m}(w)\right| \leqslant C$ for $w \in S_{j}^{\delta}, m=5,6$. Furthermore, it follows from [18, Theorem II.I] that $v_{7}(w) \in H^{1}(D)$. Thus, $v(w)$ has nontangential limits almost everywhere on $\partial D$ which define a function in $L^{1}(\partial D)$. 
Let $q_{j}=v-h_{j}$ in $D_{j}\left(2 r_{j}\right)$. Then $q_{j}$ is holomorphic in $D_{j}\left(2 r_{j}\right)$ and there exist integers $m_{j}$ such that

$$
f=\exp \left(f_{j}+q_{j}+2 \pi i m_{j}\right)
$$

defines a global holomorphic function in $D$ (cf. [5, p. 181]). It follows from our estimates that $f \in N(D)$.

Proof of Corollary. $\delta=\delta^{+}-\delta^{-}$where both $\delta^{+}$and $\delta^{-}$have finite area in $D$. Thus, by the Theorem, there exist two holomorphic functions $f_{1}$ and $f_{2}$ which define $\delta^{+}$and $\delta^{-}$respectively such that $f_{1}, f_{2} \in N(D)$ and $g=f_{1} \phi / f_{2}$, where $\phi$ has no zeros of poles in $D$.

Then $\phi=f_{2} g / f_{1}$ has finite characteristic and so is in $N(D)$, so $f_{1} \phi \in N(D)$ and $f_{1} \phi$ and $f_{2}$ have nonzero nontangential limit values almost everywhere on $\partial D$. Q.E.D.

\section{BIBLIOGRAPHY}

1. L. Gruman, Les classes de Hardy et de Nevanlinna généralisées, C. R. Acad. Sci. Paris Sér. A-B 277 (1973), A359-A361.

2. - Generalized Hardy and Nevanlinna classes (to appear).

3. G. M. Henkin, Integral representation of functions in strongly pseudoconvex regions and applications to the $\bar{\partial}$-problem, Mat. Sb. 82 (124) (1970), 300-308 = Math. USSR Sb. 11 (1970), 273-281. MR 42 \#534.

4. - Continuation of bounded holomorphic functions from submanifolds in general position to strictly pseudo-convex domain, Izv. Akad. Nauk SSSR Ser. Mat. 36 (1972), 540-567 = Math. USSR Izv. 6 (1972), 536-563. MR 46 \#7558.

5. L. Hörmander, An introduction to complex analysis in several variables, Van Nostrand, Princeton, N. J., 1966. MR 34 \#2933.

6. $\mathrm{N}$. Kerzman, Hölder and $L^{p}$ estimates for solutions of $\bar{\partial} u=f$ in strongly pseudoconvex domains, Comm. Pure Appl. Math. 24 (1971), 301-379. MR 43 \#7658.

7. G. Laville, Résolution au $\partial \bar{\partial}$ avec croissance dans des ouverts pseudoconvexes étoilés de $\mathrm{C}^{n}$, C. R. Acad. Sci. Paris Sér. A-B 274 (1972), A554-A556. MR 45 \#3760.

8. P. Lelong, Ensembles singuliers impropres des fonctions plurisousharmoniques, J. Analyse Math. 36 (1957), 263-303.

9. - Fonctions entières (n variables) et fonctions plurisousharmoniques d'ordre fini dans $\mathrm{C}^{n}$, J. Analyse Math. 12 (1964), 365-407. MR 29 \#3668.

10. - Fonctionelles analytiques et fonctions entierès ( $n$ variables), Les Presses de l'Université de Montreal, 1968.

11. - Fonctions plurisousharmoniques et formes différentielles positive, Gordon \& Breach, Paris, 1968. MR 39 \#4436.

12. G. Mueller, Functions of finite order in the ball, Thesis, University of Notre Dame, 1971.

13. $\mathrm{N}$. Ovrelid, Integral representaiton formulas and $L^{p}$-estimates for the $\bar{\partial}$-equation, Math. Scand. 29 (1971), 137-160. MR 48 \#2425.

14. E. Ramírez de Arellano, Ein Divisionsproblem und Randintegraldarstellungen in der komplexen Analysis, Math. Ann. 184 (1969/70), 172-187. MR 42 \#4767.

15. H. Skoda, Solution à croissance du second problème de Cousin dans $\mathrm{c}^{n}$, Ann. Inst. Fourier (Grenoble) 21 (1971), fasc. 1, 11-23. MR 45 \#588. 
16. E. M. Stein, Boundary behavior of holomorphic functions, Princeton Univ. Press, Princeton, N. J., 1972.

17. W. Stoll, A Bezout estimate for complete intersections, Ann. of Math. (2) 96 (1972), 361-401. MR 47 \#2091.

18. E. L. Stout, $H^{p}$-functions in strictly pseudo-convex domains, Amer. J. Math. (to appear).

DEPARTMENT OF MATHEMATICS, TULANE UNIVERSITY, NEW ORLEANS, LOUISIANA 70118 